\title{
POSTURAL CONTROL DOES NOT AFFECT PERFORMING OR LEARNING A TEMPORAL ESTIMATION TASK IN PHYSICALLY ACTIVE OLDER ADULTS
}

\author{
Flavio Henrique Bastos, Maria Teresa da Silva Pinto Marques-Dahi, \\ Fernando Garbeloto dos Santos, Ricardo Drews, and Umberto Cesar Corrêa \\ Motor Behavior Laboratory, School of Physical Education and Sport, \\ University of São Paulo, Brazil
}

Original scientific paper

https://doi.org/10.26582/k.51.1.6

\begin{abstract}
:
Evidence suggests that postural control could act as a secondary task, leading to a negative effect on sensorimotor skill learning. To investigate this issue, twenty older adults (average age $=70.5$ years, $\mathrm{SD}=5.6$ ) were distributed into two groups, according to the body position maintained during the acquisition (AQ) of a temporal estimation task: performing the task standing with feet together (STA) or sitting (SIT). During the $\mathrm{AQ}$, participants performed 90 trials of the task consisting of synchronising the arrival of two rectangles ('Target A' and 'Target B') to a target line. The velocity of Target A was chosen by the participants, among three possible ones, before each trial, without exceeding 30 trials per velocity. Target $\mathrm{B}$ had only one velocity and should be released by the participants, with a button press, when they judged it would reach the target line simultaneously with Target A. Contrary to what has been shown in studies with reaction time, postural control did not affect the performance of our temporal estimation task. Additionally, no effects on sensorimotor learning - inferred by immediate and delayed transfer tests - were found. Result suggests that postural control may not interfere with cognitive resources used to perform a simultaneous task, when this task does not demand fast processing, i.e., is not highly time constrained. Future studies should consider the physical activity level of participants, since the fact that all participants in the present study were physically active may have contributed to the observed results.
\end{abstract}

Key words: sensoriomotor skills, motor learning, aging, static posture

\section{Introduction}

Functional decline is one of the major concerns associated with the aging process. In this sense, the ability to acquire new motor skills as well as the ability to use them in new contexts (i.e., transfer) are critical to maintain adaptability during aging. Literature indicates that the ability to learn sensorimotor skills changes as we age (Seidler, 2007, 2010). Thus, knowledge about the factors that affect this ability as well as the mechanisms that underlie changes associated with aging become relevant to provide support for intervention in areas such as Physical Education and Rehabilitation.

Evidence indicates that older adults show poor performance in controlling static posture (Huxhold, Li, Schmiedek, \& Lindenberger, 2006; Maylor \& Wing, 1996; Raymakers, Samson, \& Verhaar, 2005) and use more cognitive resources associated with information processing - also named 'attentional resources' (Shumway-Cook \& Woollacott, 2006), and 'working memory' (Miyake \& Shah, 1999) for postural control, compared to younger adults (Marsh \& Geel, 2000; Redfern, Jennings, Martin, \& Furman, 2001). These negative effects on postural control have been explained mainly by the reduction in the frequency with which older adults walk and perform daily living activities (Enoka, 2008).

The need of cognitive resources for postural control suggests that more demanding postures (i.e., less stable) could negatively affect sensorimotor learning, since they could act as a secondary task, in relation to the one being learned. The negative effect of the secondary task on sensorimotor learning has been shown in previous studies with young adults (Taylor \& Thoroughman, 2007, 2008). In these studies, in addition to performing a reaching skill that should be acquired, participants were asked to simultaneously perform a task of auditory discrimination, involving identifying 
and classifying an auditory stimulus as high or low (Taylor \& Thoroughman, 2007), or performing a semantic categorization (Taylor \& Thoroughman, 2008). The authors suggested that the divided attention, although not impairing feedback control of the movement, did reduce sensorimotor learning (subsequent movement adaptation).

Studies employing reaction time tasks provide indicatives with respect to how demanding different postures could be in terms of their use of available cognitive resources (e.g., Lajoie \& Gallagher, 2004; Lajoie, Teasdale, Bard, \& Fleury, 1996; Marsh \& Geel, 2000; Redfern, et al., 2001; Teasdale \& Simoneau, 2001; van Dieën, Koppes, \& Twisk, 2010). In these studies, the reaction time task is performed simultaneously with the postural task, configuring a dual-task protocol. In this case, increases in reaction time are used to indicate a greater demand of resources to perform the postural task. A study carried out by Marsh and Geel (2000), revealed that, relative to younger women, older women needed more cognitive resources to maintain an eyes open standing posture (standing with their feet shoulderwidth apart) compared to maintaining a sitting posture. Additionally, in a study using a similar body posture (Lajoie, et al., 1996) - standing with feet together - older persons with greater probability of falling (indicating difficulties concerning their postural control) showed increased reaction time relative to older persons with less probability of falling.

Therefore, one could argue that postural control could affect sensorimotor learning in older adults, since even regular postures, as standing with feet shoulder-width apart, can demand more cognitive resources of older adults (e.g., Marsh \& Geel, 2000). Postural control, in this sense, could act as the secondary task being performed while practising a sensorimotor skill. However, even though there is evidence for (a) a negative effect of performing tasks that demand cognitive resources concurrently with the acquisition of a sensorimotor skill, and (b) that older adults use more of these resources for postural control relative to younger adults, there is no evidence that performing a concurrent postural task could negatively affect sensorimotor learning. Thus, the aim of the present study was to investigate whether the demand for postural control affects performance and/or learning of a sensorimotor skill in older adults. In the present study, two groups of older adults were asked to practice a temporal estimation task in different postural conditions: seated or standing with feet together. If postural control while standing with feet together act as the secondary task for older adults, worse performance is expected during practice (acquisition phase) and learning tests (immediate and delayed transfer tests).

\section{Methods}

\section{Participants}

Twenty volunteers (average age $=70.5$ years, $\mathrm{SD}=5.6$ ), enrolled in the Physical Education for Older Adults Course - held at the School of Physical Education and Sport of the University of São Paulo - participated in the study. All participants were submitted to an interview with an experienced Physical Educator before taking part of the Physical Education for Older Adults Course. This interview is aimed at certifying that all participants are capable of carrying out Instrumental Activities of Daily Living (Spirduso, Francis, \& MacRae, 2004), such as using basic communication skills, doing housework, preparing meals and running errands by driving or using public transportation. Presenting normal, or corrected-to-normal, vision and not having previous experience with the sensorimotor task were used as inclusion criteria. All participants gave their written informed consent, which was approved by the Ethics Committee of the School of Physical Education and Sport of the University of São Paulo (CAAE: 01281112.2.0000.5391).

\section{Task and procedures}

The equipment consisted of a computer, a LCD (Liquid Crystal Display) monitor of 22" $(120 \mathrm{~Hz}$ refresh rate, 1680x1050 resolution) and a button (switch) connected by a coaxial cable to a data acquisition card (Labjack U3-HV). A custom GNU / Octave script, using Psychtoolbox toolbox - which allows the presentation of visual stimuli and record information with a millisecond accuracy (Kleiner, Brainard, \& Pelli, 2007) - running on a Linux operating system (Ubuntu 12.04), managed the task and the data acquisition.

The task goal was to synchronise the arrival of two rectangles ('Target A' and 'Target B'), moving from left to right in the LCD monitor, to a target line (Figure 1). Participants were positioned in front of the LCD monitor (according to their group and experimental phase) and were required to press the switch with their thumb at the exact time they judged they could release Target B in order to synchronise the arrival of both targets to the target line. The task started with both targets in the same vertical position. Target A started moving between 1.5 and 3 seconds (pseudo-randomly) after the start of the task and Target B was set into motion when the participant pressed the switch. Target A could move at three possible velocities, selected by the participant after each trial, by pressing the keys 1 , 2 or 3 on a keyboard. A trial was initiated when the participant selected a velocity. At velocities 1,2 and 3, the displacement of Target A until the target line took, respectively, 2790, 2490 and $2180 \mathrm{~ms}$. In turn, to reach the target line, Target B took $1060 \mathrm{~ms}$. 


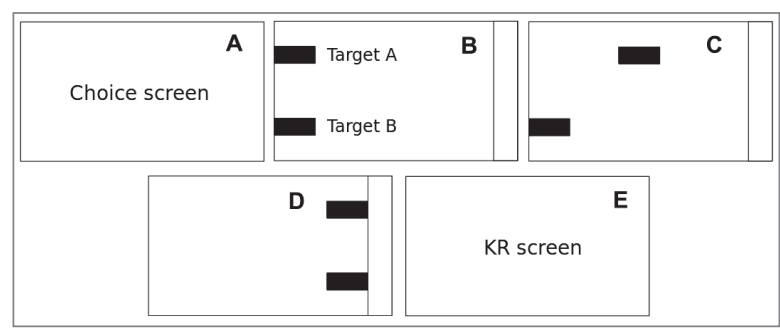

Figure 1. Schematic representation of the task (not to scale). A: screen presenting the number of trials at each velocity and the keyboard keys to select them. B: static targets before the Target A started moving with the selected velocity. C: Target A in motion. D: Ideal performance (task goal). E: Knowledge of results (KR) presentation.

This task was chosen for its minimal involvement of possible body movements that could result in perturbations to balance, since only a button press with the thumb is required as a motor response.

The experiment was composed of three phases, acquisition (AQ), immediate transfer test (ITT) and delayed transfer test (DTT). Before starting, participants were randomly assigned to two experimental groups, according to the body position they were asked to maintain during the AQ: STA (performed the task standing with their feet together) and SIT (performed the task in a sitting position).

Participants performed one trial at each velocity of Target A, before the AQ, for posterior inference about their initial performance on the task - baseline. Participants performed 90 trials of the task in the AQ, 30 trials on each of the three Target A velocities. Prior to the AQ, participants were informed about the three velocities of Target A and that they could choose any of them before each trial, not exceeding 30 trials at each velocity. Participants were also told that after those 90 trials they would take a test in which the velocities of the target would be randomly arranged. They were instructed to select the velocities, during the AQ, to prepare for this test and informed that the number of trials in each velocity would be displayed to them before each trial. A similar method was employed by Bastos, Marinovic, de Rugy and Tani (2013) to infer about learning strategies based on the choices made by participants during the AQ. After each trial participants received information about their performance in the task (knowledge of results $\mathrm{KR}$ ). KR was provided in milliseconds (ms), with no decimal places and no signal, but indicating whether Target B intercepted the line before or after Target A. KR remained on the screen until participants pressed the switch to proceed to the next screen, where they would choose the velocity of Target A for the next trial.

Started immediately after the AQ, the ITT consisted of 24 trials in which eight trials were performed for each Target A velocity, in a pseudorandom order (without consecutive velocities: 1, 2,
$1,3,2,3,1,2,3,2,1,3,1,2,1,3,2,3,1,2,3,2$, $1,3)$. Both groups, STA and SIT, performed the ITT standing with their feet together. The DTT was identical to the ITT, but occurred 15 minutes after the ITT. No KR was provided during either the ITT or DTT. When asked to keep standing with their feet together (during AQ - for STA group - ITT or DTT), participants stood on an EVA (Ethil Vinil Acetat) plate (1 $\mathrm{m} \mathrm{x} 1 \mathrm{~m} \mathrm{x} 2 \mathrm{~cm}$ thickness).

\section{Data analysis}

The data were organized and analyzed using $\mathrm{R}$, a language and environment for statistical computing (R Core Team, 2016). The significance level was set at $\alpha=.05$.

With respect to the AQ, absolute temporal error (AE), defined as the absolute value of the difference between the arrival of both targets at the target line, variable temporal error (VE), defined as the standard deviation of the temporal error over 15 trials (6 blocks of 15 trials -90 in total) and constant temporal error (CE), defined as the difference between the arrival of both targets at the target line, were the dependent measures of interest. The mean absolute, variable and constant temporal errors in the AQ were submitted to non-parametric two-way permutation tests (2 groups X 6 blocks), with blocks as a repeated measures factor, using the function 'ezPerm' in the R package 'ez. The mean absolute temporal error of the first trial performed at each velocity was used as an indicative of the participants' initial performance (baseline). The number of times participants changed the velocity being performed and the percentage of each velocity per block of trials were also considered.

The time interval participants remained with the KR on the screen (KR-time) was used as an indicative of the time used to process that information. Additionally, the time interval participants remained with the velocity choices on the screen (decision-time), before starting the next trial, was also considered. Both, the mean KR-time and the mean decision-time were submitted to non-parametric two-way permutation tests (2 groups X 6 blocks), with blocks as a repeated measures factor.

Differences among blocks of trials in the AQ were further assessed through pairwise Wilcoxon tests using the FDR (false discovery rate) correction (Benjamini \& Hochberg, 1995).

Data from both, ITT and DTT, were analyzed in two blocks of 12 trials each. The mean absolute, variable and constant temporal errors, the dependent measures of interest, were submitted to non-parametric two-way permutation tests ( 2 groups X 6 blocks), with blocks as a repeated measures factor.

Wilcoxon tests, for independent samples, were used to assess differences between the groups regarding the number of velocity switches and the baseline performance. 


\section{Results}

\section{Baseline}

No difference was found between the groups in the baseline $(\mathrm{p}=.90)$, indicating they had similar skill level before the AQ.

\section{Acquisition}

Both groups reduced the AE during the $\mathrm{AQ}$, as indicated by the main effect of blocks $(\mathrm{p}<.01)-$ Figure 2. The post-hoc comparisons did not localize the differences. The permutation test revealed no differences between the groups $(p=.961)$ or interaction between the groups and blocks $(\mathrm{p}=.136)$. The $\mathrm{VE}$ also decreased during the AQ for both groups $(p<.05)$ although differences could not be localized by the post-hoc comparisons. No main effect of the group $(p=.905)$ or interaction between the groups and blocks $(p=.22)$ were found. Figure 2 shows both groups reducing temporal anticipation along the blocks of the AQ, which was confirmed by the main effect of blocks for the $\mathrm{CE}(\mathrm{p}<.05)$. The post-hoc comparisons did not localize the differences. Furthermore, the permutation test revealed no differences between the groups $(\mathrm{p}=.982)$ or interaction between the groups and blocks $(p=.227)$ for the CE. Overall, our results indicate no effect of postural control on magnitude, variability or bias of error during the AQ.

As can be seen in Figure 3, the KR-time was reduced along the blocks of the AQ for both groups $(\mathrm{p}<.05)$, but post-hoc comparisons did not localize the differences. No main effect of the groups $(\mathrm{p}=.917)$ or interaction between the groups and blocks $(\mathrm{p}=.878)$ were found. With respect to the decision-time, no effect of blocks ( $p=.104)$, groups $(p=.107)$ or interaction $(p=.525)$ were found. These results indicate no effect of postural control on the time to decide about their practice or on the time to process KR about the temporal estimation task.

Figure 4 shows the percentage of each velocity used per block of trials during the AQ. As can be seen, participants of both groups showed a tendency of choosing the velocities by the number used to specify them. Specifically, participants tended to choose the velocities in an ascending order, with most of their trials at Velocity 1 being performed in the first two blocks of the AQ, followed by velocities 2 and 3.

\section{Immediate and delayed transfer tests}

The permutation tests revealed no differences, for any of the performance variables analyzed, concerning the immediate transfer test or the delayed transfer test (Table 1).

Permutation test (2 groups X 3 velocities) was applied to verify whether participants could

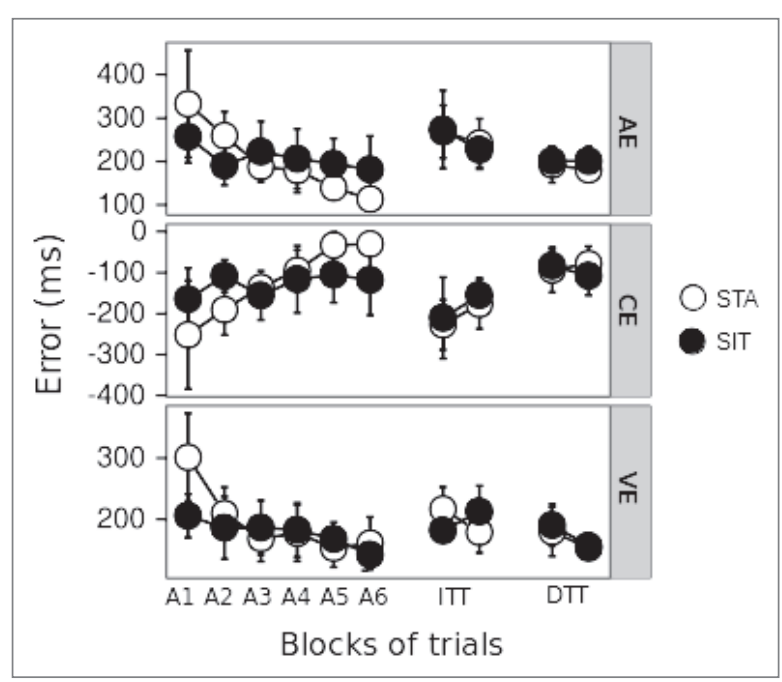

Figure 2. Absolute (AE), constant (CE) and variable (VE) errors during acquisition (A1-A6), immediate transfer test (ITT) and delayed transfer test (DTT), for both groups (STA and SIT). Error bars represent the \pm SEM

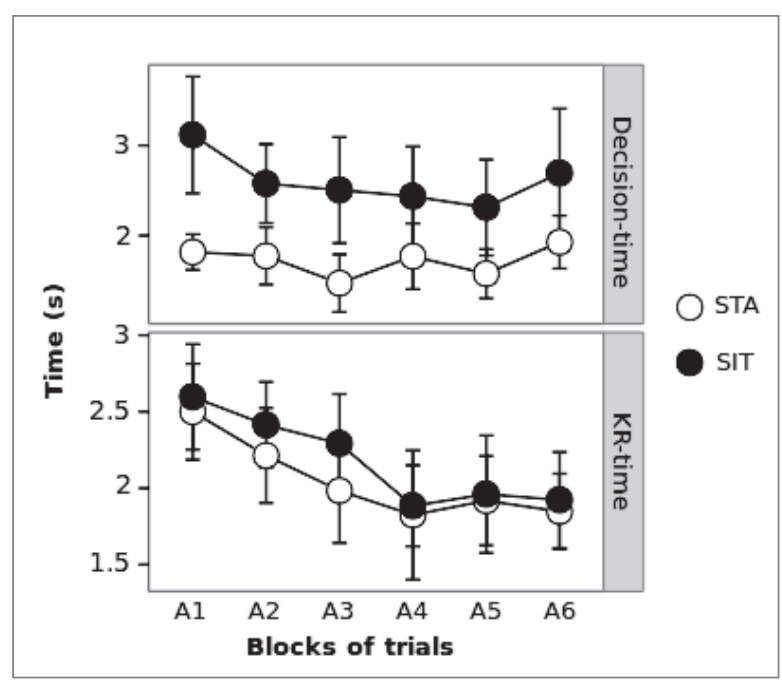

Figure 3. Average time taken to decide which velocity to perform in the next trial (decision-time) and average time knowledge of results remained on the screen (KR-time), during the acquisition (A1 - A6), for both groups (STA and SIT). Error bars represent the $\pm S E M$

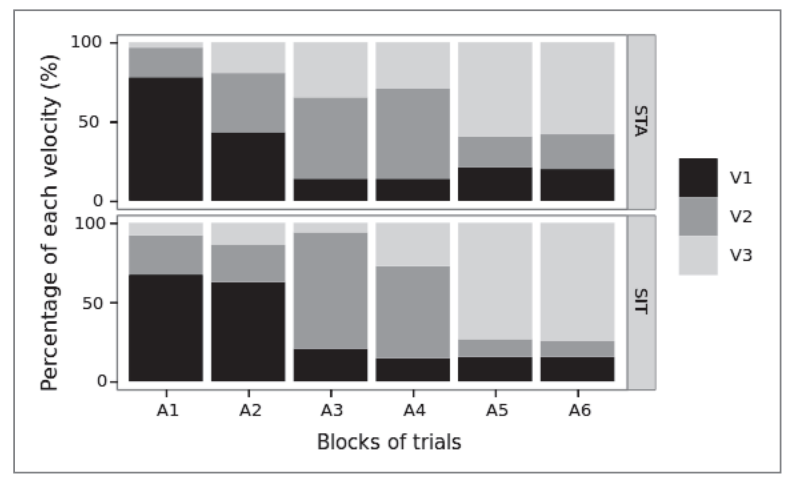

Figure 4. Percentage of each velocity (V1, V2 and V3) selected by the participants in the STA group and in the SIT group during the acquisition $(A 1-A 6)$. 
Table 1. P values of the permutation tests for each error type in ITT and DTT

\begin{tabular}{|c|c|c|c|c|c|c|}
\hline \multirow[b]{3}{*}{ Interaction } & \multicolumn{3}{|c|}{ ITT } & \multicolumn{3}{|c|}{ DTT } \\
\hline & $\mathrm{AE}$ & VE & $\mathrm{CE}$ & $\mathrm{AE}$ & VE & $\mathrm{CE}$ \\
\hline & .730 & .079 & .929 & .766 & .874 & .417 \\
\hline Group & .950 & 990 & .832 & .749 & .842 & .862 \\
\hline Block & .268 & .856 & .175 & .783 & .246 & .828 \\
\hline
\end{tabular}

have used the difficulty of the task (in an easyto-hard fashion) as a criterion to choose among velocities during the AQ. Although no effects of group $(\mathrm{p}=.986)$ or interaction $(\mathrm{p}=.82)$ were found, the performance was different among velocities, as indicated by the main effect of blocks $(\mathrm{p}<.001)$. Post-hoc comparisons revealed that the performance at Velocity 1 was different from the performance at Velocity $3(p=.02)$, with a greater error at Velocity 1 (average $=286.6, \mathrm{SD}=193.9$ ) followed by Velocity 2 (average $=214.5, \mathrm{SD}=154.4)$ and Velocity 3 (average $=152.1, \mathrm{SD}=77.7$ ).

\section{Discussion and conclusions}

The aim of the present study was to investigate whether the demand for postural control could negatively affect performance and learning of a sensorimotor skill in older adults. The rationale was based on the evidence that even everyday postures, as standing with the feet shoulder-width apart, has been shown to demand more cognitive resources of older adults (e.g., Marsh \& Geel, 2000) and, therefore, could have negative effects similar to tasks being simultaneously performed with the one being learned (Taylor \& Thoroughman, 2007, 2008).

The absence of difference between the groups in the AQ indicates that the demand for postural control required by standing with the feet together did not affect the performance in the temporal estimation task. Since this result does not corroborate studies using reaction time (e.g., Lajoie et al., 1996; Marsh \& Geel, 2000), possible explanations for the absence of difference between the groups are the different demands of each task (reaction time and temporal estimation) and the amount of trials performed. With respect to the former, Salthouse (1984) have demonstrated in a study with typists that age was only correlated with the performance when the typing task was comparable with a choice reaction time task - by presenting a reduced number of words. When participants were submitted to a normal typing task, with a wide preview window of words and, thus, allowing anticipation to contribute to performance instead of only their information processing speed, the correlation between performance and age approached zero. Based on this evidence, a possible explanation for the discrepancy between the results observed in previous studies (using reaction time) and the results observed in the present study could be that participants did not have to rely on their information processing speed to perform our temporal estimation task, as they would have to perform a reaction time task. Therefore, it is reasonable to suppose that postural control may not interfere with cognitive resources used to perform a simultaneous task when the task does not demand fast processing, i.e., in a highly time constrained context. One could argue that, besides the different demands of the tasks, our results could be explained by the greater number of trials used in this study, due to the sensorimotor learning design. However, if this was the reason for the absence of difference between the groups, a difference should have been observed in the first block of trials. Although, descriptively, the performance of the STA group was more variable in the first block of trials, relative to the performance of the SIT group, no statistically significant results were found.

The STA group was expected to show inferior performance in the learning tests (ITT and DTT), compared to the SIT group, since keep a standing position with the feet together could act as a secondary task, hindering sensorimotor learning. However, considering that both groups improved with practice and that there was no difference between them in the transfer tests, it is possible to conclude that processes associated with sensorimotor learning were not negatively affected by the postural control demanded by standing with the feet together in older adults.

Although the absence of difference between the experimental groups, both in the AQ and in the transfer tests, can be interpreted as a consequence of the low perturbation caused by the standing with the feet together position, this interpretation deserves considerations. Participants in this study were all physically active, as they regularly participated in a physical activity program. This fact cannot be neglected in explaining the results, since the reduction in the frequency with which older adults walk and perform daily living activities is one of the main explanations for negative effects on postural control (Enoka, 2008). As aging does not act at the same speed and in an identical way for all individuals (Spirduso, Francis, \& MacRae, 2004), participants with different functional levels could be affected differently by a postural task, considering the important role postural control plays in 
tests used to infer about functional levels of older adults (e.g., Rikli \& Jones, 2013). Nevertheless, this remains a question to be tackled in future studies. In addition, discriminating participants based on the quality of their postural control, as in other studies (e.g., Brauer, Woollacott, \& Shumway-Cook, 2001), could also lead to a deeper understanding of the relationship between performing, and learning, a sensorimotor skill and postural control.

Although several studies have shown that selfcontrolled practice conditions lead to gains in sensorimotor learning, a result observed especially in young adults (Sanli, Patterson, Bray, \& Lee, 2013), the present study did not aim to investigate this issue. Self-controlled practice organisation was used to allow inference about possible strategies during practice (Bastos, et al., 2013) and whether they would be affected by postural control. Specifically, groups were instructed to choose among velocities during the AQ in order to prepare for a test, so that learning strategies could be inferred by how participants chose among velocities. Results suggest that participants did not elaborate their strategies based on prior knowledge of test context - as in the study of Bastos et al. (2013) with young adults - but on the numbering that designated each velocity. Specifically, even with prior knowledge of test context, participants showed the tendency to choose velocities according to their numerical order (i.e., most trials at Velocity 1 performed in the first two blocks of trials, followed by most trials at Velocity 2 and Velocity 3). It is important to note, however, that this behaviour was not observed for all participants, as indicated by the variability in all blocks. In this sense, future studies may discriminate subgroups among participants regarding the strategies used when practice organisation is self-controlled. Furthermore, participants did not seem to have based their decisions on the difficulty of the task either. The results indicated that, for both groups, the performance at each velocity was different, with inferior performance at slower velocities. Although velocity increase is associated with increased difficulty in some tasks (e.g., Fitts, 1954), other studies using temporal estimation tasks show that it does not necessarily occur in this type of task (e.g., Bastos, et al., 2013). Thus, this result suggests that participants did not organise their practice considering the difficulty of the task, i.e. in an easy-to-hard fashion

In this report we have shown that standing with feet together does not affect performing or learning a temporal estimation task in physically active older adults. Given that a reduced frequency of older adults' walk and daily activities performance is one of the main explanations for negative effects on postural control, future studies should investigate whether the physical activity level could affect the relationship between postural control and temporal estimation performance and learning.

\section{References}

Bastos, F.H., Marinovic, W., de Rugy, A., \& Tani, G. (2013). Prior knowledge of final testing improves sensorimotor learning through self-scheduled practice. Human Movement Science, 32(1), 192-202. doi:10.1016/j.humov.2012.11.008

Benjamini, Y., \& Hochberg, Y. (1995). Controlling the false discovery rate: A practical and powerful approach to multiple testing. Journal of the Royal Statistical Society. Series B (Methodological), 57(1), 289-300.

Brauer, S.G., Woollacott, M., \& Shumway-Cook, A. (2001). The interacting effects of cognitive demand and recovery of postural stability in balance-impaired elderly persons. The Journals of Gerontology Series A: Biological Sciences and Medical Sciences, 56(8), M489-M496.

Enoka, R. (2008). Neuromechanics of human movement ( $4^{\text {th }}$ ed.). Champaign, IL: Human Kinetics.

Fitts, P.M. (1954). The information capacity of the human motor system in controlling the amplitude of movement. Journal of Experimental Psychology, 47(6), 381-391. doi:10.1037/h0055392

Huxhold, O., Li, S.-C., Schmiedek, F., \& Lindenberger, U. (2006). Dual-tasking postural control: Aging and the effects of cognitive demand in conjunction with focus of attention. Brain Research Bulletin, 69(3), $294-305$. doi:10.1016/j.brainresbull.2006.01.002

Kleiner, M., Brainard, D.H., \& Pelli, D.G. (2007). What's new in Psychtoolbox-3? A free cross-platform toolkit for Psychophysics with Matlab \& GNU/Octave. Perception, 36. (ECVP Abstract Supplement).

Lajoie, Y., \& Gallagher, S.P. (2004). Predicting falls within the elderly community: Comparison of postural sway, reaction time, the Berg balance scale and the Activities-specific Balance Confidence (ABC) scale for comparing fallers and non-fallers. Archives of Gerontology and Geriatrics, 38(1), 11-26.

Lajoie, Y., Teasdale, N., Bard, C., \& Fleury, M. (1996). Upright standing and gait: Are there changes in attentional requirements related to normal aging? Experimental Aging Research, 22(2), 185-198. doi:10.1080/03610739608254006

Marsh, A.P., \& Geel, S.E. (2000). The effect of age on the attentional demands of postural control. Gait \& Posture, 12(2), 105-113. doi:10.1016/S0966-6362(00)00074-6 
Maylor, E.A., \& Wing, A.M. (1996). Age differences in postural stability are increased by additional cognitive demands. The Journals of Gerontology. Series B, Psychological Sciences and Social Sciences, 51(3), P143-P154.

Miyake, A., \& Shah, P. (1999). Models of working memory: Mechanisms of active maintenance and executive control. Cambridge University Press.

R Core Team. (2016). R: A language and environment for statistical computing (Version 3.3.2). Vienna, Austria: R Foundation for Statistical Computing.

Raymakers, J.A., Samson, M.M., \& Verhaar, H.J.J. (2005). The assessment of body sway and the choice of the stability parameter(s). Gait and Posture, 21(1), 48-58. doi:10.1016/j.gaitpost.2003.11.006

Redfern, M.S., Jennings, J.R., Martin, C., \& Furman, J.M. (2001). Attention influences sensory integration for postural control in older adults. Gait and Posture, 14(3), 211-216.

Rikli, R.E., \& Jones, C.J. (2013). Development and validation of criterion-referenced clinically relevant fitness standards for maintaining physical independence in later years. The Gerontologist, 53(2), 255-267.

Salthouse, T.A. (1984). Effects of age and skill in typing. Journal of Experimental Psychology: General, 113(3), $345-371$. doi:10.1037/0096-3445.113.3.345

Sanli, E.A., Patterson, J.T., Bray, S.R., \& Lee, T.D. (2013). Understanding self-controlled motor learning protocols through the self-determination theory. Frontiers in Psychology, 3, 611. doi:10.3389/fpsyg.2012.00611

Seidler, R.D. (2007). Aging affects motor learning but not savings at transfer of learning. Learning and Memory, 14(12), 17-21. doi:10.1101/1m.394707

Seidler, R.D. (2010). Neural correlates of motor learning, transfer of learning, and learning to learn. Exercise and Sport Sciences Reviews, 38(1), 3.

Shumway-Cook, A., \& Woollacott, M.H. (2006). Motor control: Translating research into clinical practice ( $3^{\text {rd }}$ revised ed.). Philadelphia: Lippincott Williams and Wilkins.

Spirduso, W., Francis, K., \& MacRae, P. (2004). Physical dimensions of aging (2 ${ }^{\text {nd }}$ ed.). Champaign, IL: Human Kinetics.

Taylor, J.A., \& Thoroughman, K.A. (2007). Divided attention impairs human motor adaptation but not feedback control. Journal of Neurophysiology, 98(1), 317-326. doi:10.1152/jn.01070.2006

Taylor, J.A., \& Thoroughman, K.A. (2008). Motor adaptation scaled by the difficulty of a secondary cognitive task. PLoS One, 3(6), e2485.

Teasdale, N., \& Simoneau, M. (2001). Attentional demands for postural control: The effects of aging and sensory reintegration. Gait and Posture, 14(3), 203-210. doi:10.1016/S0966-6362(01)00134-5

van Dieën, J.H., Koppes, L.L.J., \& Twisk, J.W.R. (2010). Postural sway parameters in seated balancing; their reliability and relationship with balancing performance. Gait and Posture, 31(1), 42-46. doi:10.1016/j.gaitpost.2009.08.242

Submitted: February 7, 2017

Accepted: April 16, 2018

Published Online First: August 31, 2018

Correspondence to:

Flavio Henrique Bastos, Ph.D.

Laboratório de Comportamento Motor, Escola de

Educação Física e Esporte

Universidade de São Paulo

Av. Prof. Mello Moraes, 65, Cidade Universitária, São

Paulo, Brazil

Zip Code: 05508- 030

Fax: +551138153342

Phone: +55 1130912147

E-mail: bastosfh@usp.br 